\title{
Introduction à l'œuvre du Mauricien Léoville L'Homme à partir des notes introductives au poème Le Rock de Cirné
}

\section{Robert Furlong}

\section{Q OpenEdition}

1 Journals

\section{Édition électronique}

URL : https://journals.openedition.org/coma/670

DOI : $10.4000 /$ coma.670

ISSN : 2275-1742

\section{Éditeur}

Institut des textes \& manuscrits modernes (ITEM)

\section{Référence électronique}

Robert Furlong, «Introduction à l'œuvre du Mauricien Léoville L'Homme à partir des notes introductives au poème Le Rock de Cirné », Continents manuscrits [En ligne], 6 | 2016, mis en ligne le 31 octobre 2016, consulté le 12 juillet 2022. URL : http://journals.openedition.org/coma/670 ; DOI : https://doi.org/10.4000/coma.670

Ce document a été généré automatiquement le 12 juillet 2022.

Creative Commons - Attribution - Pas d'Utilisation Commerciale - Pas de Modification 4.0 International - CC BY-NC-ND 4.0

https://creativecommons.org/licenses/by-nc-nd/4.0/ 


\title{
Introduction à l'œuvre du Mauricien Léoville L'Homme à partir des notes introductives au poème Le Rock de Cirné
}

\author{
Robert Furlong
}

1 Dans son édition de novembre 1921, la revue littéraire mauricienne L'Essor ${ }^{1}$ inaugure la publication de fragments d'un poème inachevé du Mauricien Léoville L'Homme intitulé Le Rock de Cirné. Ces fragments, au nombre de six, sont publiés dans les livraisons mensuelles successives de cette revue de novembre 1921 à avril 1922. Le premier fragment est précédé, en guise de notes introductives, de cinq courts paragraphes de l'auteur qui y commente son projet d'écriture en qualifiant ce texte de " prologue d'un poème ambitieux [qu'il] commençait il y a une vingtaine d'années. » Et il poursuit en ces termes: "Je plaçais, en notre île, l'action de ce poème, dont l'idée me vint à la lecture d'une délicieuse légende hindoue où figure l'oiseau Rock habitant l'Himalaya, oiseau banni au-delà de l'équateur par un religieux pour avoir enlevé une fiancée le jour de ses noces. Je faisais de l'oiseau fabuleux l'enveloppe d'une âme qui avait vécu plusieurs existences en des formes diverses. [...] Sa forme de Rock était sa dernière incarnation. Il était venu cacher la fiancée hindoue dans une des montagnes de notre île, alors inconnue, et environ cent ans avant qu'elle fut découverte et nommée Cirné par le navigateur Mascareignes. »

2 Le manuscrit en prose de ce projet poétique sous la forme de deux petits cahiers atteste en effet que la première rédaction date de 1894 et que celle-ci fut modifiée en 1895 et 1896. Mais compte tenu des différences dans la graphie de l'auteur, il est certain que d'autres révisions difficiles à dater intervinrent plus tard. 
Fig. 1 : Page 11 du manuscrit du poème Le Rock de Cirné

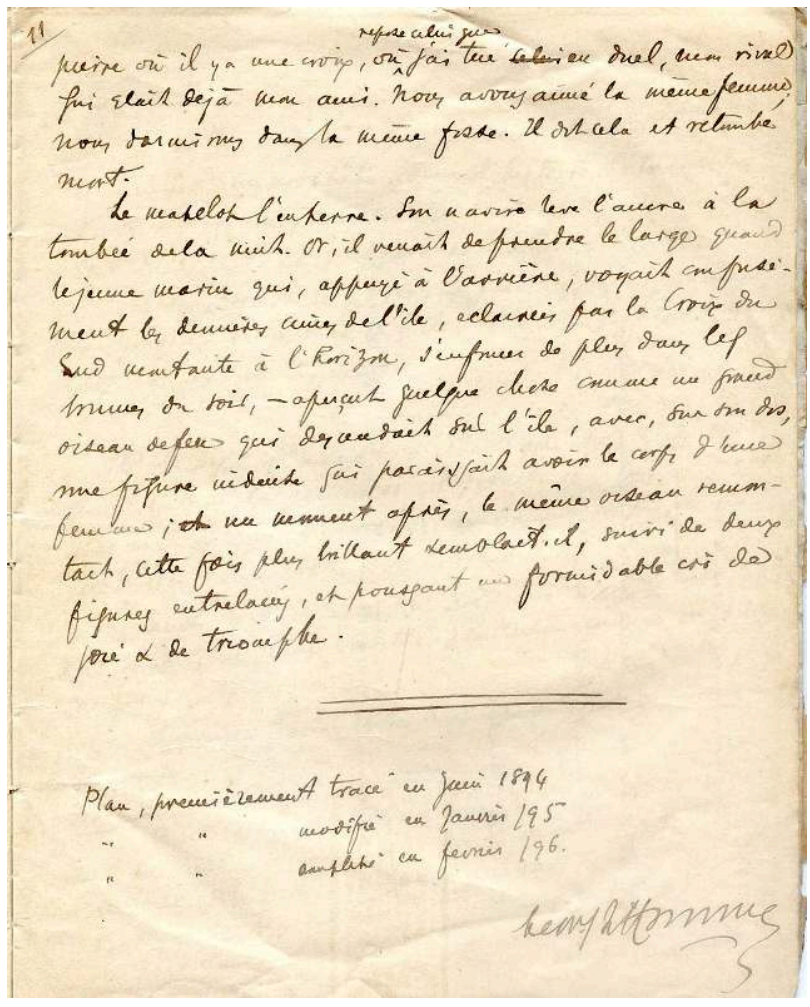

Archive personnelle de Robert Furlong

Le premier cahier comporte sur onze pages la trame résumée quasiment sans ratures et d'une écriture régulière comme en témoigne le fac-similé de la onzième page portant la signature de l'auteur et les dates évoquées plus haut. Des numéros portés sur ces pages renvoient à une succession de compléments et de corrections développée sur une trentaine de pages supplémentaires parmi lesquelles des pages annulées, des passages biffés, des mots rayés et des insertions diverses. Par exemple, la rencontre entre un marin naufragé du nom de Pedro ${ }^{2}$ et l'oiseau Rock $^{3}$ est plusieurs fois réécrite ; la date de naissance du naufragé est d'abord 1298, puis 1402; l'action se passe entre les années 1383 et 1385, dates corrigées pour 1422 à 1424 ; le lieu du naufrage de ce marin est modifié pour être finalement proche du lieu où la Virginie de Bernardin de SaintPierre meurt dans le naufrage du Saint-Géran ${ }^{4}$; les lieux où demeure l'oiseau à Maurice sont également plusieurs fois modifiés... Quant au personnage devenu l'oiseau Rock, des couches successives d'évocations de sa personnalité s'accumulent au fur et à mesure du manuscrit. Il est d'abord, avant Jésus-Christ, un prophète de Dieu respecté mais qui perd sa respectabilité en devenant l'amant d'une Égyptienne qui le convertit à l'idolâtrie. Il est tué par un lion et Satan prend possession de son âme en le faisant habiter dans le corps d'un oiseau Rock. C'est sous cette forme qu'il enlève le jour de ses noces une jeune hindoue qu'il a vu nue dans son bain et qu'il est banni de l'hémisphère Nord. Cherchant un havre désert dans le Sud pour cacher sa captive, il choisit l'île Maurice. Par la suite, le poète envisage de donner à l'oiseau Rock des qualités de visionnaire afin qu'il évoque des faits et des personnalités importantes de l'histoire future de l'île : le gouverneur français Mahé de La Bourdonnais qui est le fondateur de la capitale et des bases économiques de l'île au milieu du XVIII siècle, les amours de Paul et Virginie telles que Bernardin de Saint-Pierre les raconte, la prise de l'île par les 
Anglais en décembre 1810, le tribun défenseur des droits de la population de couleur Rémy Ollier qui crée le quotidien La Sentinelle de Maurice à cette fin en 1843, le prêtre missionnaire Jacques Désiré Laval qui se dévoue de 1841 à 1864 pour les anciens esclaves laissés pour compte... Une indication biffée concerne l'esclavage : «parler de l'esclavage qui souillera l'île » avait écrit Léoville L'Homme dans son projet initial.

Le deuxième cahier est celui de la recherche documentaire que l'auteur a considéré nécessaire au traitement du sujet. Léoville L'Homme relit le Voyage à l'ssle de France ${ }^{5} \mathrm{de}$ Bernardin de Saint-Pierre et cite l'édition utilisée : Édition Aimé Martin, chez Lefèvre, Paris, 1833. Il en extrait diverses notes et observations : sur les oiseaux frégates, paillesen-cul, sur la montagne Pieter-Both qui ressemble «à la statue d'une femme ", sur la couleur des couches de roche, sur la description de certains arbres et arbrisseaux, sur les bruits de la forêt tels "le croassement des perroquets", sur les baleines, sur les ouragans et leurs manifestations, entre autres... Il relit également les Études 4 et 5 de l'ouvrage Les Harmonies de la nature ${ }^{6}$ du même auteur et en recopie des pages entières ayant trait à la faune et la flore de l'île telles que Bernardin de Saint-Pierre a pu les observer lorsqu'il séjourna dans l'île de 1768 à $1770 .$. Léoville L'Homme se réfère également à un livret en date de 1899- en fait, presque un tirage pour correction vu les imperfections qu'il contient- du statuaire Prosper d'Épinay d'ascendance mauricienne et intitulé L'̂́le du Cygne (Cirné) - L'oiseau Ruc ${ }^{7}$ d'Épinay s'étend longuement sur l'oiseau Ruc tel que décrit par Marco Polo dans ses mémoires de 1735 et complète la légende en établissant un lien avec le récit d'un géographe arabe du XIve siècle, al-Dimashqi, qui, en 1325, «dans la description de l'île de Khomor (...) parle longuement d'un oiseau Rokh et de ses cufs grands comme des coupoles" et rappelle également cette légende arabe selon laquelle un "immense oiseau » a été " banni dans les îles désertes inaccessibles et lointaines de l'hémisphère Sud pour le punir d'avoir enlevé le jour de ses noces une jeune fille revêtue de ses habits de fiancée et belle comme le jour8." "Alors bibliothécaire de la ville de Port-Louis, Léoville L'Homme avait découvert et annoté ce livret.

5 Si tout ce travail préparatoire étalé sur de si nombreuses années ne put aboutir, Le Rock de Cirné est bien « un poème ambitieux » comme le qualifie Léoville L'Homme. Terminé et publié, il aurait contribué, pour la première fois dans la littérature locale, à donner à l'île une dimension légendaire, voire mythique. Repousser le début officiel de l'histoire de l'île de quelques siècles constitue, en effet, une réécriture significative de son histoire. Et faire de cette île le refuge d'un oiseau légendaire gigantesque évoqué, d'une part, par Marco Polo dans ses mémoires et, d'autre part, par un géographe spécialiste des routes maritimes arabes est une façon d'intégrer l'île à une tradition plurimillénaire dépassant la classique subordination des auteurs insulaires de l'époque à l'Europe du XVIII ${ }^{\mathrm{e}}$ siècle en permettant un enracinement dans l'universel. Cela aurait correspondu, alors, à la création d'un mythe littéraire local, qu'on aurait pu appeler la mythologie de Cirné. En ce sens, que la jeune fiancée cachée dans le roc de la montagne soit d'origine hindoue n'est guère innocent littérairement et culturellement parlant compte tenu du métissage français-pondichérien dont Léoville L'Homme est issu.

On ne peut que regretter que Léoville L'Homme n'ait pu mener à terme cette fresque poétique. Les fragments publiés dans L'Essor sont comme suit : un Prologue de 140 vers raconte l'arrivée du marin Pedro dans l'île; un deuxième fragment intitulé L'île est déserte de 150 vers raconte l'exploration du littoral; un troisième de 200 vers détaille l'exploration de l'intérieur de l'île sous le titre L'excursion; un quatrième, Le Rock dans l'ouragan, raconte en 200 vers un impressionnant cyclone et la situation critique de 
l'oiseau Rock, que Pedro rencontre pour la première fois, piégé par un arbre immense qui l'avait blessé en lui tombant dessus et le maintenait prisonnier; un cinquième de 350 vers et intitulé Le prophète maudit est composé d'un long récit par le Rock de sa vie et de ses péripéties; un sixième, enfin, est intitulé La femme et l'arbre et constitue une fin plutôt bâclée à cette histoire. Mais on peut raisonnablement penser qu'au moment où le symbolisme se développait et gagnait progressivement les rives mauriciennes avec ses configurations poétiques nouvelles, rédiger une telle proposition mythique en alexandrins était peut-être une erreur stratégique, si tant est qu'un poète soit un être de stratégie...

7 Si ce projet ne put se concrétiser, la carrière de Léoville L'Homme ne fut pas pour autant un échec. À l'Île Maurice, Léoville L'Homme, né en 1857 et décédé en 1928, a acquis cette dimension monumentale qui sied aux grands de la nation. Plusieurs rues de villes importantes portent son nom. Au Jardin de la Compagnie de Port-Louis, vaste jardin aux arbres exotiques au milieu de la capitale administrative et économique, un buste en bronze du poète, auquel une muse tend une couronne de lauriers, placé sur une haute stèle en granit au bout de l'allée principale a été installé en 1931. Léoville L'Homme traversait ce même jardin au moins deux fois par jour, et ce pendant plus de cinquante ans, lorsqu'il se rendait de la gare de chemin de fer de Port-Louis à ses bureaux de journaliste, puis de bibliothécaire municipal. Au pied de cet hommage érigé par la municipalité de Port-Louis et inauguré le 27 décembre 1931, un livre en bronze est ouvert et le texte d'un poème intitulé Lettre à un ami est offert à l'appréciation de tous.

8 Par-delà la statue, l'homme. Une enfance aux Salines, quartier de bord de mer à proximité de la capitale, où il est né en 1857. Un père journaliste qui écrivit le premier traité de féminisme à Maurice en $1880^{\circ}$. Une famille aux moyens modestes ce qui, sans doute, explique que Léoville sera retiré de l'école à l'âge de 15 ans pour commencer à gagner sa vie comme ouvrier typographe. La légende (ou la vérité ?) veut que le jeune Léoville, qui parlait mal le français, eut un jour à rétablir les rimes d'une romance : il s'en serait si bien tiré qu'il fut définitivement contaminé par le virus de la versification et de la poésie. 
Fig. 2 : Léoville L'Homme à 22 ans

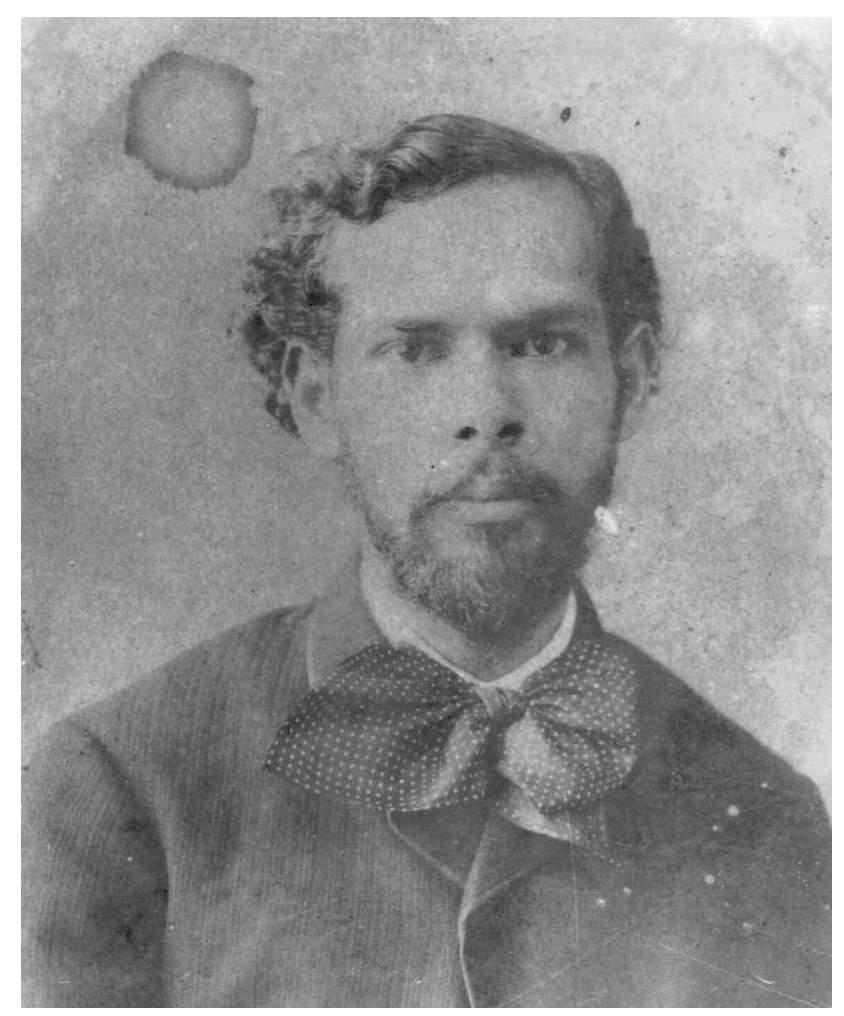

Archive personnelle de Robert Furlong

9 À 24 ans, il entre comme rédacteur à La Sentinelle de Maurice, important journal libéral fondé par Rémy Ollier, le héraut cité plus haut de la bourgeoisie métisse en quête de reconnaissance. Léoville L'Homme dirigera le journal à partir de 1883 avant de le quitter sur un désaccord quant à la ligne politique choisie. Le débat qui divisait alors l'île a un rapport direct avec la toute première consultation électorale qui a lieu en 1886. Quittant La Sentinelle avec panache, Léoville L'Homme fonde avec son père en 1885 le quotidien Le Droit qu'il dirige jusqu'au début de 1887, avant de lancer La Presse Nouvelle fin 1887 et de créer La Défense qui vécut de 1897 à 1900. C'était des années de braise où quasiment chaque édition des six ou sept grands journaux paraissant alors en quotidien ou en hebdomadaire était le théâtre de prises de position fortes et passionnées. L'Île Maurice vivait alors - dans ces années 1880-1900 - une période cruciale de son évolution politique où conservateurs et réformistes s'affrontaient par journaux interposés.

10 Après sa nomination comme bibliothécaire de la municipalité de Port-Louis en 1903, dégagé du poids et des contraintes de la «politique politicienne » et du journalisme au quotidien, Léoville L'Homme put se consacrer davantage à l'écriture. Des notes manuscrites en préparation d'une conférence sur le journalisme résument sa conviction finale sur la presse : «Ce qui y prédomine, c'est le fait, surtout le fait. Un réalisme égoïste encore que général. Le moins de réflexion et d'analyse qu'il est possible. Le fait triomphant partout, l'analyse n'est plus de saison. » Le seul organe d'information qu'il accepta, par la suite, de porter sur les fonds baptismaux et de diriger fut Mauritiana, une revue historique et littéraire bimensuelle de haut niveau dont il a été le rédacteur en chef de 1908 à 1911. Il collabore sous forme d'articles et de 
chroniques à plusieurs revues littéraires dont L'Essor mais aussi Le Voleur Mauricien, Les Roses de Noël, Le Soleil de Juillet...

11 Par-delà le journaliste pris dans les affrontements partisans, il y a le poète. Sa première plaquette, Pages en vers ${ }^{10}$ contenant deux poèmes d'inspiration biblique, date de 1882 , elle est suivie en 1883 d'une seconde plaquette intitulée Les Étoiles ${ }^{11}$ composée d'un unique poème fortement imprégné de convictions chrétiennes et de références bibliques. Son unique pièce de théâtre, la seule qu'il écrira d'ailleurs tant elle fut rejetée par le public, paraît la même année...Vinrent ensuite, le recueil Poèmes païens et bibliques $^{12}$ de 1887 contenant une quinzaine de poèmes sur des personnages de la bible ou d'autres sources, voire religions (Aphrodite, Artemis, Ekelmia, Khadidja ...) et marqués par un classicisme teinté de sensualité, voire d'érotisme léger. Les larmes de Sainte Scholastique ${ }^{13}$ en 1892 confirme la propension de Léoville L'Homme à puiser sa thématique dans le religieux, Sainte Scholastique ayant créé dans les années 500 le premier monastère bénédictin féminin. Ce poème sera repris en 1897 dans le recueil Poésies diverses ${ }^{14}$ avec quatre autres textes d'inspiration plus légère donnant place à des fées, à une berceuse pour enfants et à un admirable coucher de soleil. Ce recueuil marque une évolution de son inspiration moins assujettie aux divinités chrétiennes et une ouverture vers l'Inde immortelle et le Nirvâna. Pages en vers ${ }^{15}$ en 1905 n'est pas une réédition de sa première plaquette, mais un recueil abondant et éclectique tant par les formes poétiques que l'inspiration : vingt-deux poèmes, parmi lesquels une réécriture de quelques textes antérieurs notamment Eve dont le manuscrit est conservé. Poèmes épars ${ }^{16}$ est l'unique recueil qui parait à Paris. Le dernier recueil, Poésies et poèmes ${ }^{17}$, date de 1926, soit deux ans avant le décès du poète. Composé de trente-trois textes, un peu plus d'un tiers, soit quatorze, sont des reprises remaniées de textes parus dans Pages en vers de 1905. Par ailleurs, il publie régulièrement dans la presse et les revues littéraires des poèmes qui ne sont pas repris en recueil. Léoville L'Homme avait vers la fin de sa vie un projet de recueil déjà fort élaboré. Il en avait même déjà retenu le titre : Les Voix $d u \operatorname{larg} e^{18}$. Ce recueil devait comprendre quarante-quatre poèmes et l'ordre dans lequel ils allaient figurer dans la plaquette était déjà établi. Selon le décompte fait de la main même de Léoville L'Homme, l'ensemble de ces textes représentait 2769 vers. Par ailleurs, tous les poèmes devant figurer dans ce recueil étaient prêts et certains d'entre eux avaient déjà paru dans la revue littéraire L'Essor.

12 Au journaliste et au poète s'ajoutent le chroniqueur et le conteur. Dès 1884, il adopte, pour ce type d'écrits, un pseudonyme : Léon Lauret. Tant dans La Sentinelle de Maurice que dans les colonnes du quotidien Le Radical (sous le titre générique Feuillets de Carnet de 1908 à 1918) et dans celles du Mauricien (sous le titre générique de Feuilles au vent de 1918 et 1922), puis dans ces deux derniers quotidiens jusqu'à sa mort en 1928, il publie régulièrement des chroniques sur des sujets divers et variés. Sous ce même pseudonyme et pendant cette même période, il signe de nombreux contes publiés dans diverses revues littéraires.

13 Une chose est certaine : Léoville L'Homme fut le premier écrivain mauricien à laisser une œuvre abondante. Outre les recueils, contes et chroniques publiés, il existe également des manuscrits inédits constituant des archives. Celles-ci ne sont malheureusement pas publiques et sont la propriété d'un membre de la famille. Parmi ces textes, quelques projets de poèmes comme celui devant célébrer la montagne du Morne Brabant, un lieu ayant servi de refuge aux esclaves marrons, ou La Clarinette, 
Fig. 3 : Projet de poème La Clarinette

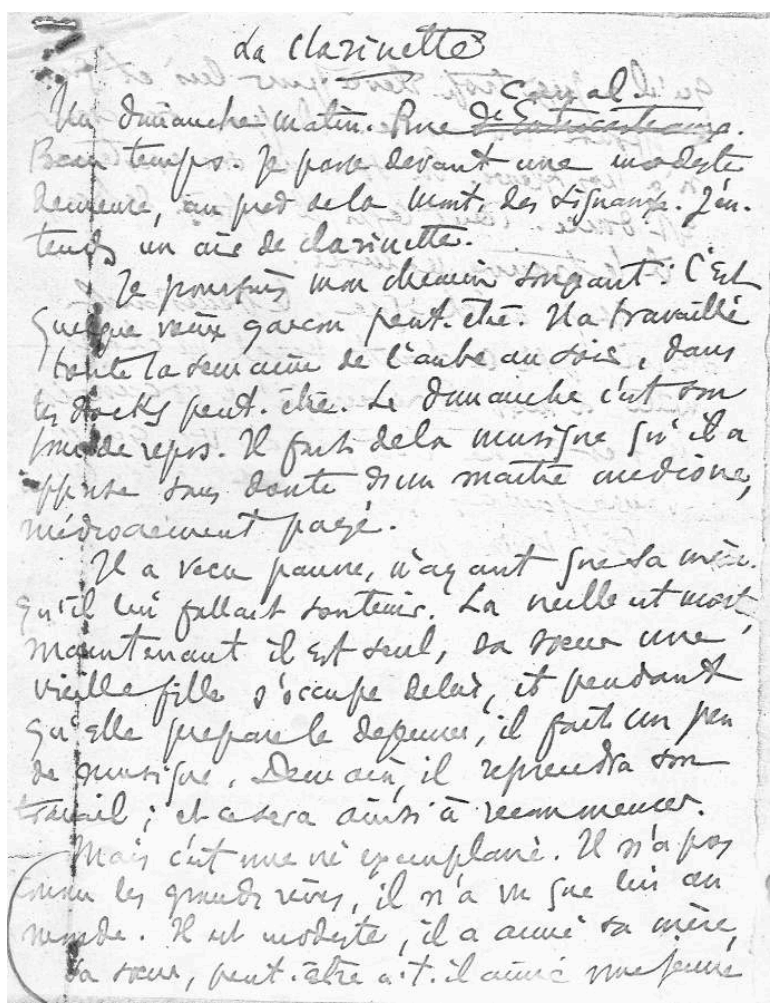

Archive personnelle de Robert Furlong

daté du dimanche 18 mai 1913 et devant être écrit "en vers libres »; des manuscrits datés au nombre de trente, certains ayant été publiés dans la presse ou en recueil à l'instar du Moine Piter-Both écrit en 1907 
Fig. 4 : Manuscrit du poème Le Moine Piterboth

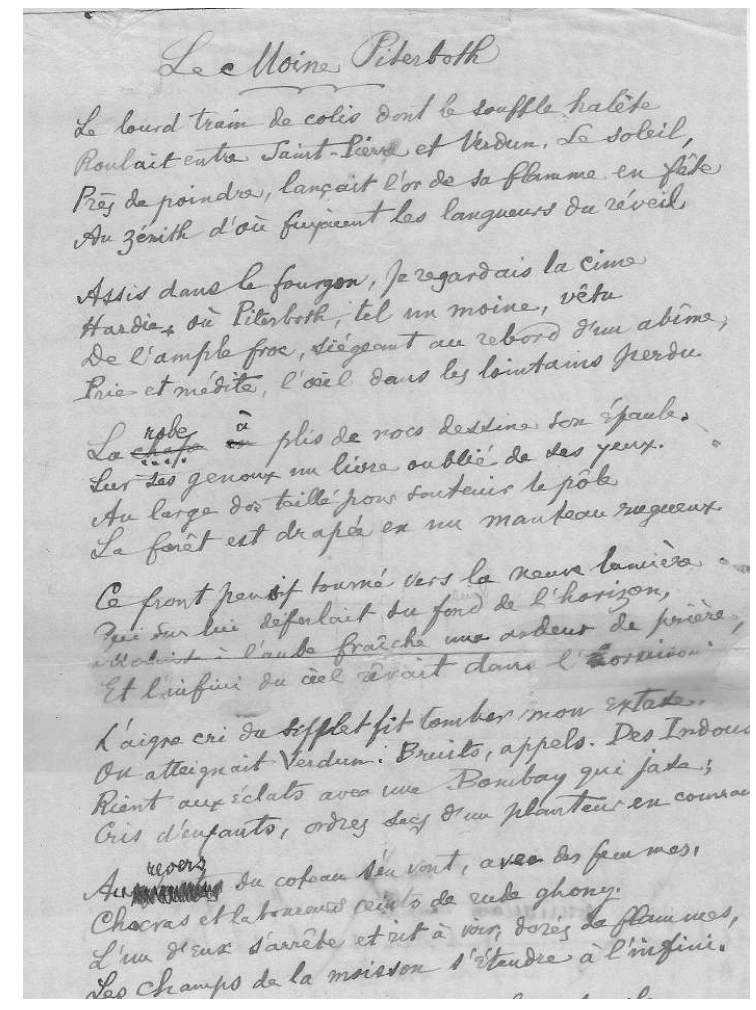

Archive personnelle de Robert Furlong

et paru en 1927 dans la revue L'Essor et d'autres n'ayant jamais été publiés tel La Case du Sorcier de août 1902 
Fig. 5 : Manuscrit du poème La case du sorcier

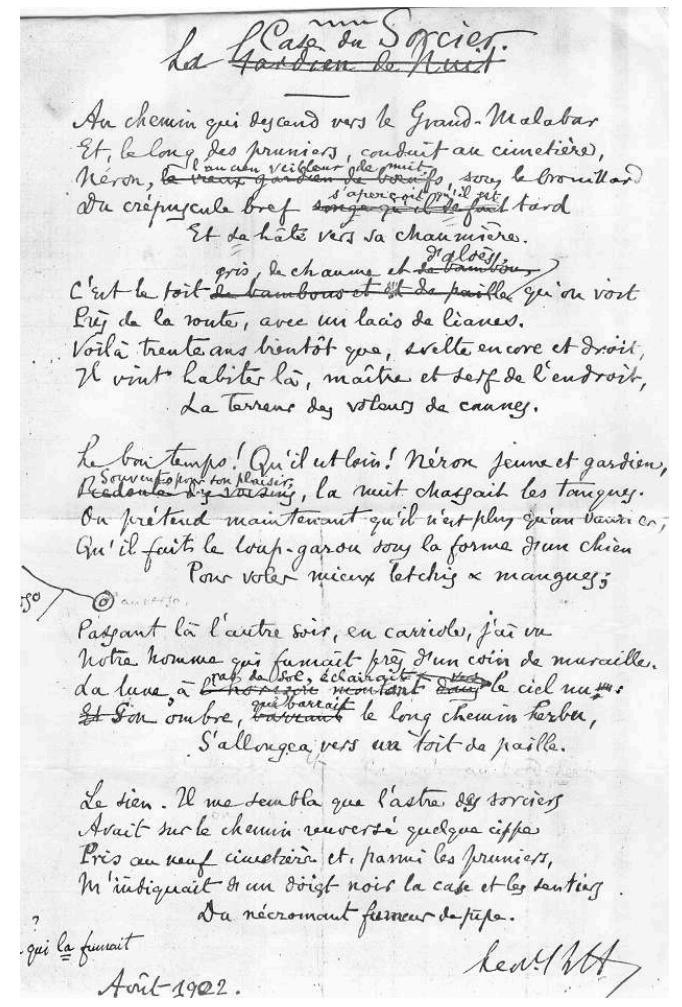

Archive personnelle de Robert Furlong

des manuscrits non datés au nombre de vingt-huit et des fragments rassemblés sous le titre Vers détachés pouvant servir. 
Fig. 6 : Vers détachés pouvant servir, couverture

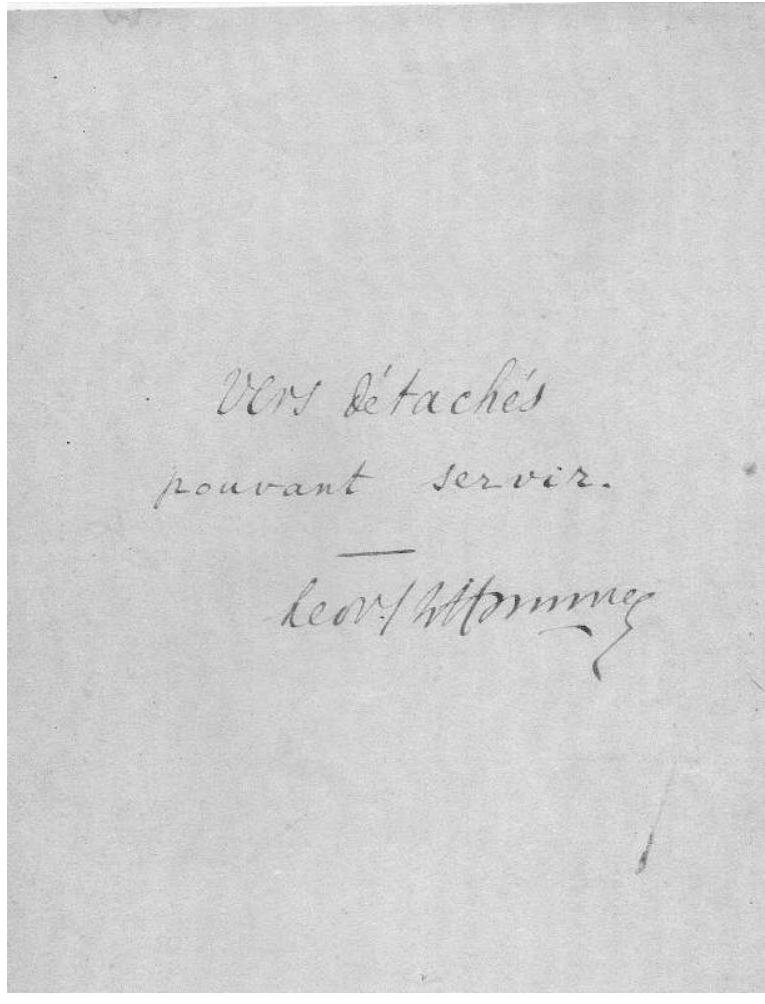

Archive personnelle de Robert Furlong

14 Y figurent également les brouillons de quelques discours et ses notes de conférences sur la littérature ${ }^{19}$ où il affirme, par exemple, que «la poésie n'est pas seulement une question d'orfèvrerie et un poète n'est pas avant tout ou seulement un ciseleur ; il doit être un peintre, un évocateur d'images, un remueur de sentiments, un enchasseur d'idées.» Ou encore "Si la raison, si le cœur n'ont pas inspiré les sentiments de l'auteur, son œuvre ne peut être assurée que d'une mort proche ou lointaine, mais de toute façon certaine. » Plusieurs poèmes portent en sous-titre des indications qui font penser qu'ils appartiennent à des regroupements: "Poèmes d'Orient ", "Poèmes mauriciens », comme Malaria ... 
Fig. 7.a : Manuscrit du poème Malaria

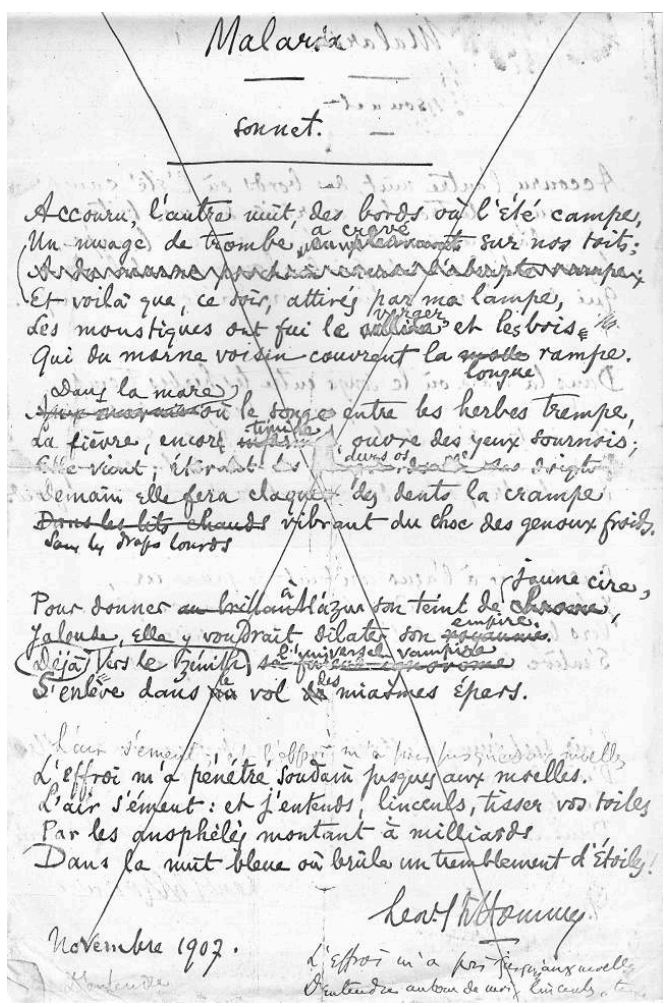

Archive personnelle de Robert Furlong

Fig. 7.b : Manuscrit du poème Malaria

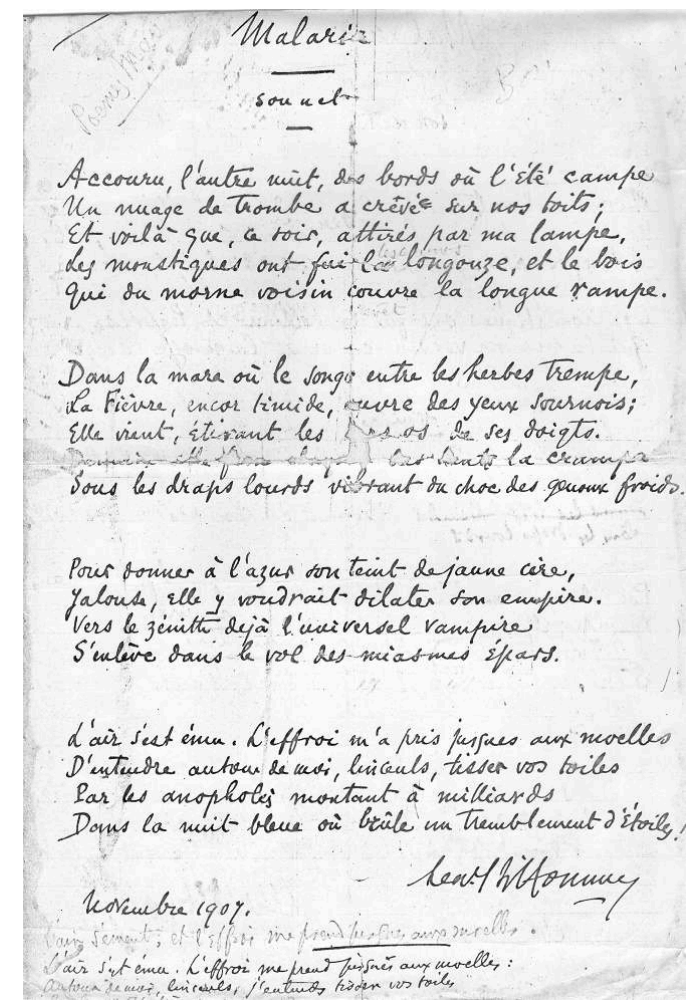

Archive personnelle de Robert Furlong 
Cette liasse de manuscrits, y compris les cahiers manuscrits du poème Le Rock de Cirné, rassemble autant de documents nécessitant une analyse approfondie et pouvant donner des informations nouvelles quant à l'écriture de Léoville L'Homme. Celui-ci est toujours considéré comme «le père de la littérature mauricienne. »

\section{NOTES}

1. La revue littéraire L'Essor a été l'organe du Cercle littéraire de Port-Louis. Créée en 1919, elle a paru pendant près de quarante ans et a joué un rôle déterminant dans l'évolution de la littérature mauricienne en offrant un espace d'expression régulier aux écrivains en organisant sur une base annuelle un concours littéraire.

2. Comme le navigateur portugais Pedro Mascarenhas qui donna son nom à l'archipel d'îles du sud-ouest de l'océan Indien dont Maurice fait partie.

3. Souvent orthographié Roc dans le manuscrit.

4. Dans Paul et Virginie, roman de Bernardin de Saint-Pierre publié en 1788, Virginie meurt noyée lors du naufrage du bateau le Saint Géran la ramenant à l'Isle de France, nom que portait l'île Maurice pendant sa période française de 1715 à 1810.

5. Saint-Pierre, Bernardin de, Voyage à l'Isle de France, à l'Isle de Bourbon et au Cap de BonneEspérance, par un officier du Roi, Merlin, Amsterdam et Paris, 1773, 2 volumes.

6. Paris : Méquignon-Marvis, 1815. Publié de façon posthume.

7. Épinay, Prosper d', L'T̂le du Cygne (Cirné)-L'oiseau Ruc, extrait d'un ouvrage inédit, Port-Louis, typ. Du Cernéen, 1899.

8. Citation extraite du livret de Prosper d'Épinay.

9. L'Homme, Pierre, La Femme, ses droits et ses devoirs, Port-Louis, Imprimerie de la Sentinelle de Maurice, $1880.210 \mathrm{p}$.

10. L'Homme, Léoville, Pages en vers, Port-Louis, General Steam Publishing Company, 1882. 36 p.

11. L'Homme, Léoville, Les étoiles, Port-Louis, imprimerie du Mercantile Record, 1883.14 p.

12. L'Homme, Léoville, Poèmes païens et bibliques, Port-Louis, nouvelle Imprimerie Dupuy, 1887. $94 \mathrm{p}$.

13. L'Homme, Léoville, Les larmes de Sainte Scholastique, Port-Louis, imprimerie du Journal de Maurice, 1892.

14. L'Homme, Léoville, Poésies diverses, Port-Louis, imprimerie de The Planters and Commercial Gazette, 1897. 17 p.

15. L'Homme, Léoville, Pages en vers, édition remaniée et augmentée, Port-Louis, chez l'auteur, 1905. $96 \mathrm{p}$.

16. L'Homme, Léoville, Poèmes épars, Paris, Éd. Jouve, $91 \mathrm{p}$.

17. L'Homme, Léoville, Poésies et poèmes, Port-Louis, librairie Esclapon, 1926. 142 p.

18. Un feuillet manuscrit comporte toutes les informations à ce sujet.

19. Les conférences publiques qu'il animait régulièrement sur la littérature et sur l'art d'écrire étaient réputées et attiraient les intellectuels et écrivains de cette époque. 


\section{RÉSUMÉS}

Léoville L'Homme est une figure emblématique de la littérature mauricienne et sa contribution à l'évolution de cette production littéraire est remarquable tant en qualité qu'en volume. Il a été rédacteur en chef d'importants quotidiens qu'il a créés pour certains d'entre eux, journaliste militant en faveur de réformes constitutionnelles, chroniqueur dans plusieurs quotidiens, conteur régulièrement publié dans les revues littéraires locales et poète adulé : son apport a laissé une forte empreinte, lui-même devenant un modèle et ce, même si un de ses projets poétiques majeurs, Le Rock de Cirné, a été un échec.

Léoville L'Homme is an emblematic figure of Mauritian literature and his contribution to its development is remarkable both in quality and volume. He was the editor-in-chief of important daily newspapers, some of which he created, a politically active journalist in favour of constitutional reforms, a columnist in several dailies and an admired poet: his input has left a deep lasting impression, becoming himself a model in spite of the failure of one of his major poetical projects, Le Rock de Cirné.

\section{INDEX}

Mots-clés : Léoville L'Homme, Maurice

\section{AUTEUR}

\section{ROBERT FURLONG}

Robert Furlong, chercheur associé à l'ITEM-CNRS, est spécialiste de la littérature mauricienne de langues française et créole (Malcolm de Chazal, Léoville L'Homme, René Noyau, Marcel Cabon, entre autres). Ses publications comprennent notamment : plusieurs inédits de Malcolm de Chazal ainsi que des introductions et articles critiques sur Chazal dont il a présidé la fondation de 2011 à 2014 ; un Panorama de la littérature mauricienne. La production créolophone (2007) ; une intégrale René Noyau, l'œuvre (en 4 volumes, 2012) ; une anthologie En revues et en français. Une anthologie de nouvelles, chroniques et contes mauriciens (2015) retraçant la dynamique des revues littéraires à Maurice. Robert Furlong est également comédien et a animé plusieurs émissions littéraires à la télévision mauricienne. Officier dans l'Ordre des Arts et des Lettres, il préside depuis 2020 le Centre Culturel d'expression française de Maurice. 